\title{
Design of a prospective, multicenter, and cohort study of an innovative electromagnetic navigation bronchoscopy in diagnosing pulmonary nodules among Chinese population
}

\author{
Fangfang Xie ${ }^{1,2,3}$, Jian Zhang $^{4}$, Liming Cao ${ }^{5}$, Xiaoxuan Zheng ${ }^{1,2,3}$, Junxiang Chen ${ }^{1,2,3}$, Ying Li ${ }^{1,2,3}$, \\ Baohui Han ${ }^{2}$, Jiayuan Sun ${ }^{1,2,3}$ \\ ${ }^{1}$ Department of Respiratory Endoscopy, ${ }^{2}$ Department of Pulmonary Medicine, Shanghai Chest Hospital, Shanghai Jiao Tong University, Shanghai \\ 200030, China; ${ }^{3}$ Shanghai Engineering Research Center of Respiratory Endoscopy, Shanghai 200030, China; ${ }^{4}$ Department of Respiratory Medicine, \\ Xijing Hospital, Air Force Medical University, Xi'an 710032, China; ${ }^{5}$ Department of Respiratory Medicine, Xiangya Hospital of Central South \\ University, Changsha 410008, China \\ Contributions: (I) Conception and design: F Xie, J Sun; (II) Administrative support: J Sun, B Han; (III) Provision of study materials or patients: J \\ Sun, J Zhang, L Cao; (IV) Collection and assembly of data: F Xie, X Zheng, J Chen, Y Li; (V) Data analysis and interpretation: F Xie, J Sun; (VI) \\ Manuscript writing: All authors; (VII) Final approval of manuscript: All authors. \\ Correspondence to: Jiayuan Sun. Department of Respiratory Endoscopy, Department of Pulmonary Medicine, Shanghai Chest Hospital, Shanghai Jiao \\ Tong University, 241 West Huaihai Road, Shanghai 200030, China. Email: xkyyjysun@163.com.
}

\begin{abstract}
Electromagnetic navigation bronchoscopy (ENB) has been demonstrated to increase the diagnostic yield of peripheral pulmonary lesions. We designed two prospective clinical trials using an innovative ENB system that can combine with both thick and thin bronchoscope, including a randomized controlled clinical study to assess the usefulness of the ENB system and a real-world study to explore the optimal way to use this system. Patients with peripheral pulmonary nodules (PPNs) suspicious of lung cancer with the long diameter more than $8 \mathrm{~mm}$ and no more than $30 \mathrm{~mm}$ will be enrolled in the study. A total of 400 patients will be enrolled in the randomized controlled clinical study and randomly divided into two groups, endobronchial ultrasound (EBUS) combined with guide sheath (GS) with or without ENB. The realworld study is a single arm and observational study, at least 750 subjects will be enrolled in the study. ENB combined with or without other guided bronchoscopy techniques, fluoroscopy, and rapid on-site cytologic evaluation (ROSE) will be performed according to the judgment of the operator. The primary endpoint of the two studies is the diagnostic yield of ENB in diagnosing PPNs. The secondary endpoint includes the diagnostic yields for malignant and benign diseases, navigation time, time for finding lesions, total operation time, complication rate, etc. Enrollment for the study began in July 2018 and is currently in progress. With up to 1,150 subjects estimated to be enrolled, the study will provide evidence on the usage of the novel ENB system in diagnosing PPNs.
\end{abstract}

Keywords: Electromagnetic navigation bronchoscopy (ENB); lung cancer; peripheral pulmonary nodule (PPN); diagnosis

Submitted Nov 02, 2019. Accepted for publication Dec 03, 2019.

doi: $10.21037 /$ jtd.2019.11.75

View this article at: http://dx.doi.org/10.21037/jtd.2019.11.75

(c) Journal of Thoracic Disease. All rights reserved. 


\section{Introduction}

Lung cancer is the leading cause of cancer mortality worldwide, with an estimated 1.8 million new cases and 1.6 million deaths worldwide each year (1). In China, an estimated 733.3 thousand new lung cancer cases and 610.2 thousand lung cancer deaths occurred in 2015 (2). The National Lung Cancer Screening Trial demonstrated a reduction in lung cancer mortality with low dose computed tomography (CT) screening (3), which is widely used to detect early-stage lung cancer, leading to increasing nodules expected to be detected and waited for diagnosis.

Both American College of Chest Physicians and Asia guidelines for the evaluation of pulmonary nodules recommend non-surgical or surgical biopsy for nodules with the long diameter of 8 to $30 \mathrm{~mm}(4,5)$. Current non-surgical biopsy modalities typically include transbronchial lung biopsy (TBLB) and transthoracic needle aspiration (TTNA). Conventional TBLB is taken by using the bronchoscope for lung lesions biopsy, which has been shown to be effective in sampling peripheral pulmonary lesions (PPLs) (6). TTNA is useful for the diagnosis of PPLs with a high diagnostic yield. However, TTNA is associated with a higher rate of pneumothorax compared with bronchoscopic procedures and has the risk of metastasis implantation $(7,8)$.

With the development of imaging and guidance technologies, such as radial probe endobronchial ultrasound (R-EBUS) and electromagnetic navigation bronchoscopy (ENB), new developed technologies have been proven to improve the performance of TBLB in many studies (9-12). ENB is a technology providing guidance in diagnosing PPLs, which utilizes the electromagnetic field combined with the sensor probe to assist the endobronchial accessories in reaching the target airway. A recent meta-analysis has revealed that the overall diagnostic accuracy of ENB is $73.9 \%$, and sensitivity detecting cancer is $71.1 \%$ (10). It is recommended in patients with PPLs difficult to reach with conventional bronchoscopy (8). The first commercial ENB system was invented by superDimension (superDimension/ Bronchus system; Medtronic, Minneapolis, MN, USA), with a locatable sensor probe $(1.9 \mathrm{~mm})$ and extended working channel (EWC, $2.6 \mathrm{~mm}$ ) (13), which required a bronchoscope with a large diameter of the working channel. However, this limits bronchial insertion, which has poor visibility of peripheral small bronchi, and this may be one of the reasons why ENB has a low diagnostic yield for PPLs (14).
Given these limitations, an innovative ENB system (LungCare navigation system; LungCare Medical Technologies Ltd., Inc, Suzhou, China) has been developed to deal with these problems. They developed different size of locatable wire (LW), which can be used in combination with thick bronchoscope as well as thin or ultrathin bronchoscope (4.0, 3.0, or $2.8 \mathrm{~mm}$ of the outer diameter) that is now widely used for the diagnosis of PPLs. This real-time ENB with different guide sheath (GS) for TBLB (ENB-GS-TBLB) has been proven to be a novel method to increase diagnostic yield of PPLs with $87.18 \%$ in our initial experience (15). LungCare navigation system has been approved for sales by the China Food and Drug Administration since June 2016. We have designed two post-marketing clinical trials to assess the diagnostic yield and safety of the innovative electromagnetic navigation system for peripheral pulmonary nodules (PPNs).

\section{Methods}

\section{Study population}

We design two clinical trials, including a randomized controlled clinical study (study one) and a real-world study (study two), to assess the performance of the ENB among Chinese population. Patients meeting the following inclusion criteria and exclusion criteria will be enrolled in the two studies. Inclusion criteria are (I) patients older than 18-year-old, (II) patients with PPNs suspicious of lung cancer with the long diameter more than $8 \mathrm{~mm}$ and no more than $30 \mathrm{~mm}$ that need to obtain tissue samples for definitive diagnoses, (III) patients without bronchoscopy contraindications, (IV) patients providing written informed consent. Exclusion criteria are (I) presence of concomitant endobronchial lesion during the bronchoscopy procedure, (II) severe cardiopulmonary dysfunction and other indications that can't receive bronchoscopy. Patients with nodules of pure ground-glass opacity (GGO) or nodules without bronchus leading to or adjacent to will be excluded in study one. Three centers, including the coordinating center of Shanghai Chest Hospital, and two participating centers of Xijing Hospital of Air Force Medical University, Xiangya Hospital of Central South University, will participate in study one. To verify the performance of the innovative ENB, a real-world study (study two) will be carried out as well including at least five centers throughout China. All participants will provide written informed consent and investigators will explain the study to qualified 
A

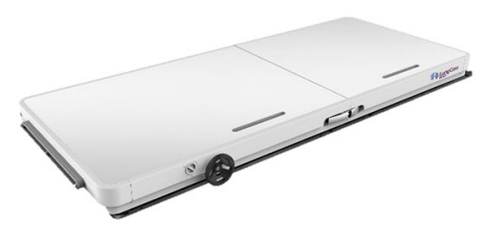

B

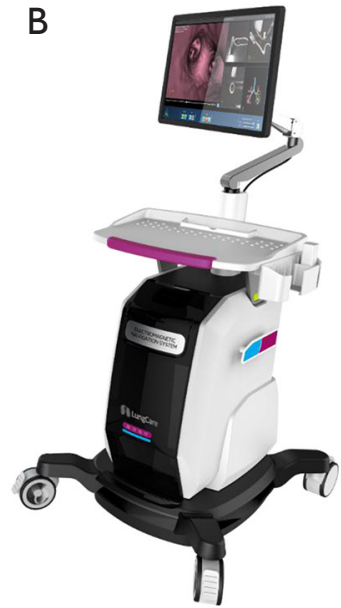

C

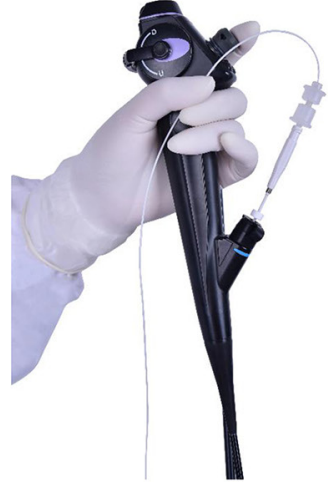

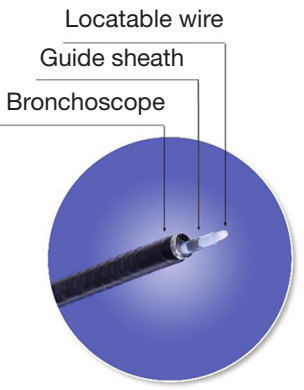

Figure 1 LungCare navigation system combined with bronchoscope. (A) Electromagnetic location board, (B) navigation system, (C) tip of LW, GS and the bronchoscope. LW, locatable wire; GS, guide sheath.

subjects prior to obtaining consent. Follow-up of each patient in this study is expected to be at least half a year since the initial ENB procedure.

\section{Test device}

The real-time ENB system (LungCare navigation system) will be used to conduct all ENB procedures. The ENB system consists of three components, including an electromagnetic location board, navigation system and LW (Figure 1). The navigation system can depict the real-time position of the $L W$ inside the electromagnetic field generated by the electromagnetic location board, providing guidance of its advance to the target airway. There are three kinds of LWs with outer diameters of $1.15,1.45$ and $1.95 \mathrm{~mm}$, which can combine with different size of the GS (SG-200C and SG-201C with the outer diameter of 1.95 and $2.55 \mathrm{~mm}$, respectively) and bronchoscope. The LW with the diameter of 1.15 and $1.45 \mathrm{~mm}$ matched with LungCare system are much thinner than the locatable guide (LG) matched with superDimension, which makes it possible that ENB combines with thinner bronchoscope to go deeper into the airway.

\section{Study design}

Two clinical studies are designed to evaluate the performance of LungCare ENB system in diagnosing PPNs. The protocols have been approved by local ethics committee (KS1829) and were registered under
ClinicalTrials.gov (NCT03569306 and NCT03716284). Patients eligible for study one will be randomly divided into two groups, ENB-EBUS-GS-TBLB group and EBUS-GS-TBLB group. The study sample will be selected using a combination of the stratified random sampling and quota methods. Stratification will be made by lesion size in the longest diameter on the CT (8-20, 20-30 mm), nodule location (inner, intermediate, peripheral) and the bronchus sign (leading to, adjacent to). Study two is a single arm study and ENB will be performed in every case. Other guided bronchoscopy techniques, sampling instruments, fluoroscopy, different types of LW and bronchoscope, or rapid on-site cytologic evaluation (ROSE) will be used according to the judgment of the operator based on the characteristics of the nodule. Study design of the two studies are presented in Figures 2,3, respectively. Patient demographics, lesion characteristics, procedure and follow-up data to be collected in the two studies are listed in Table 1. Extra data to be collected in study two are presented in Table 2. These two post-marketing studies are intended to explore the diagnostic yield of the innovative ENB system and capture clinical outcomes related to the different usage of ENB procedures.

\section{Outcomes}

Tables 3,4 describe the primary and secondary endpoints of the two studies. In addition to the primary and secondary endpoints listed above, all adverse events related to the 


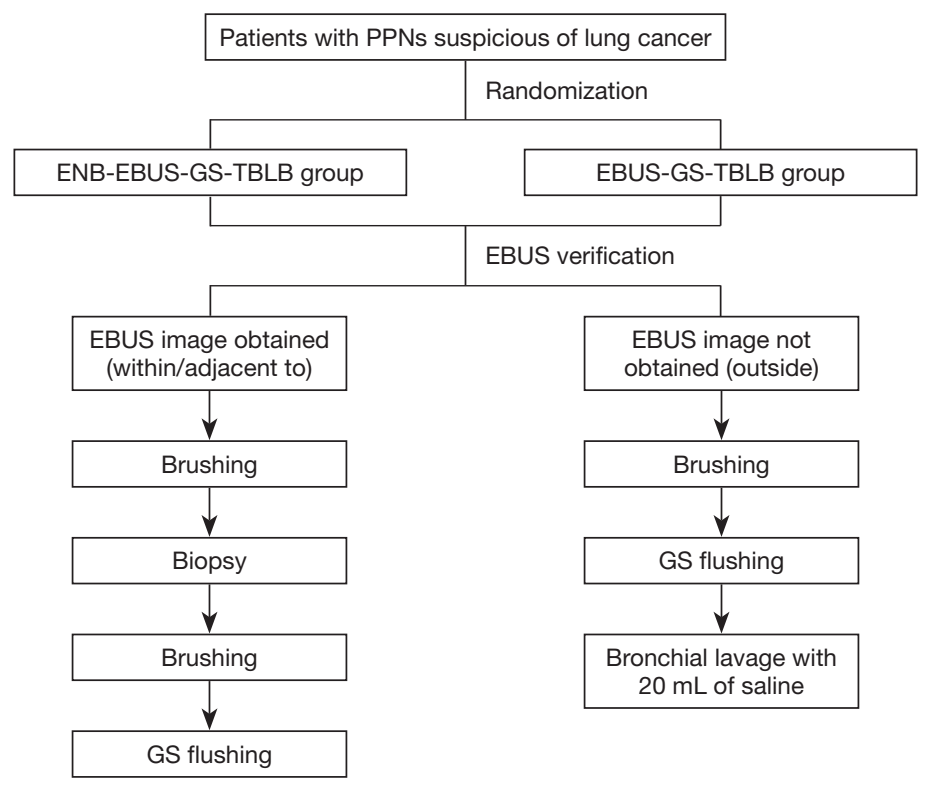

Figure 2 Flow chart of the randomized controlled clinical study. Patients with PPNs suspicious of lung cancer eligible for the criteria will be randomized into ENB-EBUS-GS-TBLB group and EBUS-GS-TBLB group. R-EBUS will be used to verify the location of the nodule. Sampling will be carried out following the sequence of brushing, biopsy, brushing and GS flushing in nodules with R-EBUS image obtained. While brushing, GS flushing and bronchial lavage with $20 \mathrm{~mL}$ of saline will be performed in nodules invisible with R-EBUS. PPN, peripheral pulmonary nodule; ENB, electromagnetic navigation bronchoscopy; GS, guide sheath; TBLB, transbronchial lung biopsy; R-EBUS, radial probe endobronchial ultrasound.

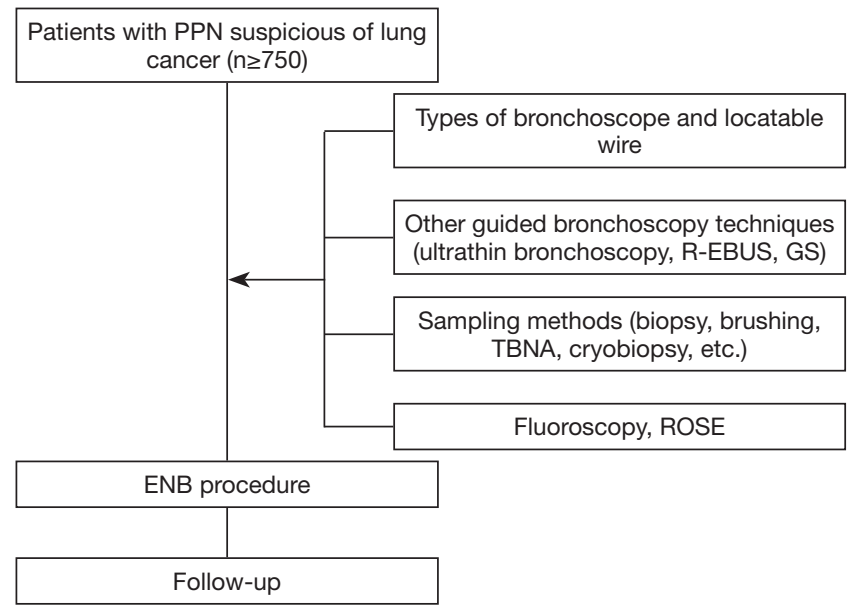

Figure 3 Flow chart of the real-world study. Patients with PPNs suspicious of lung cancer eligible for the criteria will be enrolled in the study. Different types of bronchoscope and LW, other guided bronchoscopy techniques, sampling instruments, fluoroscopy or ROSE will be used according to the judgment of the examiner based on the characteristics of the lesion. PPN, peripheral pulmonary nodule; LW, locatable wire; ROSE, rapid on-site cytologic evaluation; R-EBUS, radial probe endobronchial ultrasound; TBNA, transbronchial needle aspiration; ENB, electromagnetic navigation bronchoscopy; GS, guide sheath.
ENB procedure or associated tools will be captured and reported, including all incidences of death, pneumothorax, bronchopulmonary hemorrhage, and respiratory failure, during or post the procedure.

\section{Procedures}

\section{ENB procedure}

ENB procedure will be performed per product instructions and the institution's standard practice using the LungCare navigation system with an internal $L W$ with a diameter of $1.45 \mathrm{~mm}$ in study one and any size in study two. In the planning phase, thin-section CT data with standard format is imported into the software and is used to generate a three-dimensional CT roadmap. White light bronchoscopy is performed before registration and navigation phase to exclude concomitant endobronchial lesions. ENB procedure is performed the same as superDimension system (16). The navigation software shows four viewing panes during the navigation procedure. The $\mathrm{LW}$ will be inserted into the GS beforehand, and the GS-covered LW will be introduced via the bronchoscopic working channel and navigated to access the pulmonary nodule. The LW will be withdrawn when 
Table 1 Data to be collected in the two studies

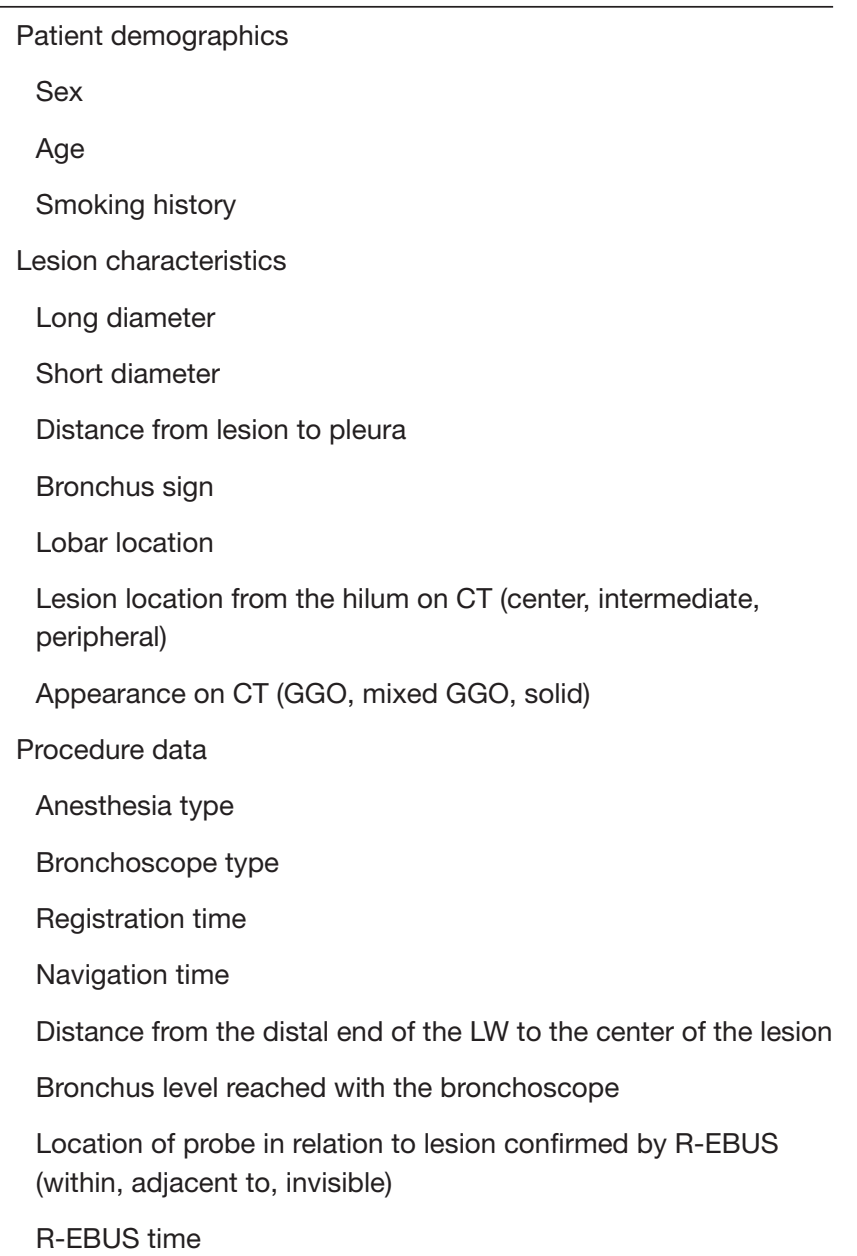

Total operation time (from the bronchoscope through the glottis to withdrawing the bronchoscope)

Number of biopsy

Number of valid biopsy visible with naked eyes

Follow-up data

Histological, cytological or microbiological results of bronchoscopy

All other diagnostic procedures (including imaging, repeat biopsy via bronchoscopy, TTNA, surgery, and so on) and diagnoses since the initial ENB procedure

All therapeutic related to lung health since last visit

Final diagnosis based on all the results

Safety data

Adverse event, action taken, relationship to the procedure, and outcome during or post the procedure

CT, computed tomography; GGO, ground-glass opacity; LW, locatable wire; R-EBUS, radial probe endobronchial ultrasound; TTNA, transthoracic needle aspiration; ENB, electromagnetic navigation bronchoscopy.
Table 2 Extra data to be collected in the real-world study

\begin{tabular}{l} 
Lesion characteristics \\
Proportion of ground glass composition (<25\%, 25-50\%, \\
$50-75 \%,>75 \%)$ \\
Procedure data \\
GS type \\
LW type \\
R-EBUS type (if applicable) \\
Visibility on fluoroscopy and fluoroscopy time (if applicable) \\
TBNA for peripheral lung lesions and the needle type (if \\
applicable) \\
Number of cryobiopsy, cryobiopsy probe type and cryobiopsy \\
time (if applicable) \\
Results of ROSE (if applicable) \\
Total sampling time \\
\hline GS, guide sheath; LW, locatable wire; R-EBUS, radial probe \\
endobronchial ultrasound; TBNA, transbronchial needle \\
aspiration; ROSE, rapid on-site cytologic evaluation.
\end{tabular}

Table 3 Primary and secondary endpoints of the randomized controlled clinical study

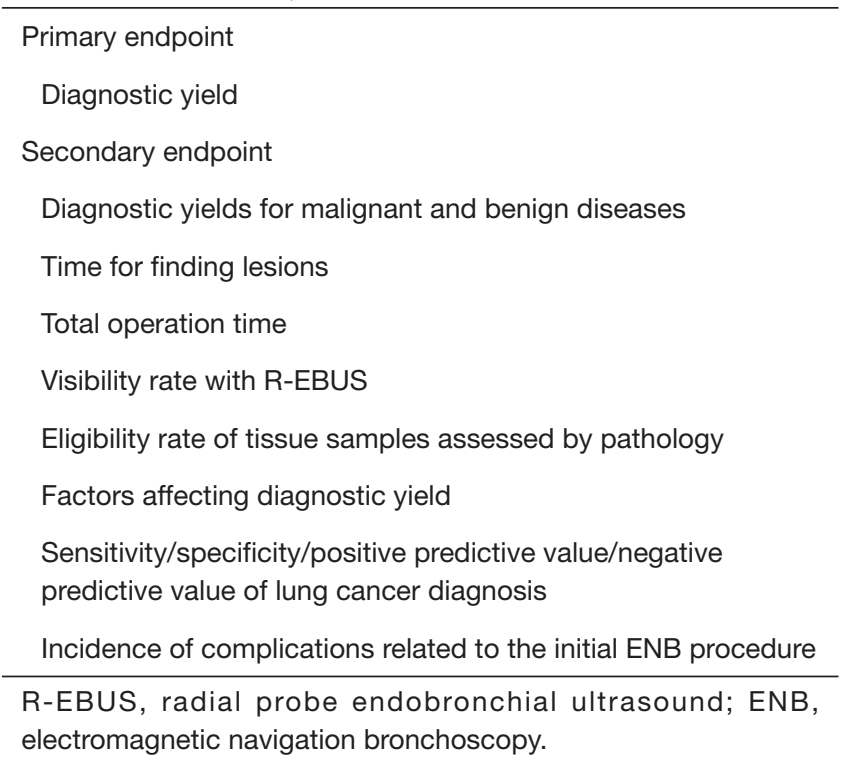

it reaches the nodule and the GS is kept in situ. Sampling instruments will be introduced into the nodule via the GS to obtain samples.

Extra procedures of study one

R-EBUS will be used both in ENB-EBUS-GS-TBLB 
Table 4 Primary and secondary endpoints of the real-world study

Primary endpoint
Diagnostic yield
Secondary endpoint
Diagnostic yields for malignant and benign diseases
Navigation time
Time for finding lesions
Total operation time
Visibility rate with R-EBUS
Eligibility rate of tissue samples assessed by pathology
Factors affecting diagnostic yield
Sensitivity/specificity/positive predictive value/negative
predictive value of lung cancer diagnosis
Usage rate of fluoroscopy
Diagnostic yields of different guided bronchoscopy techniques
Incidence of complications related to initial ENB procedure

R-EBUS, radial probe endobronchial ultrasound; ENB, electromagnetic navigation bronchoscopy.

group and EBUS-GS-TBLB group in study one. EBUS-GS-TBLB will be conducted based on the thin-section CT without ENB. All procedures will be performed without ROSE. At least five biopsy samples visible with naked eyes, two brushing samples and one flushing sample through the GS will be obtained for pathological examination. Specimens will be processed for microbiologic assessment, if necessary, according to the bronchoscopist's judgment.

\section{Extra procedures of study two}

ENB will be performed in every case in study two. R-EBUS, GS, fluoroscopy, type of the LW, type of the bronchoscope, sampling instruments or ROSE will be used according to the judgment of the operator on the basis of the characteristics of the nodule. Here, we show a representative case of study two (Figure 4).

\section{Follow-up}

All subjects will be followed-up for at least half a year, including any invasive diagnostic procedures, imaging and clinical follow-up, after the initial ENB procedure. The final diagnosis will be made by the results of the pathology and the follow-up.

\section{Data management system}

All the planning, procedure and follow-up data will be uploaded to the electronic case report form, which should be finished after the procedure or follow-up. The sponsor will check these forms to ensure the validity and authenticity of the data.

\section{Sample size}

Study one is designed to prove superiority of the diagnostic yield of ENB-EBUS-GS-TBLB group and EBUS-GSTBLB group with $80 \%$ and $65 \%$, respectively, based on published reports and our experience $(10,15,17,18)$. Demonstration of superiority with a statistical power of $80 \%$ at a one-sided significance level of 0.05 and $\delta=0.03$ would require 167 patients in each group, and we will arrange to enroll 400 patients with 200 in each group to account for dropouts $20 \%$. Although no sample size calculation is conducted for study two, at least 750 subjects will be enrolled in the study.

\section{Statistics}

Summary of the results will be presented as standard descriptive statistics. Categorical variables will be presented as percentage and continuous variables will be presented as mean, standard deviation, or median. Comparisons between groups of categorical data will be performed using Fisher's exact or Pearson's chi-squared test. Continuous variables will be assessed for normality in distribution and $t$-test or Wilcoxon rank sum test will be used. All $\mathrm{P}$ values will be two-sided. A P value of $<0.05$ indicates statistical significance. All data will be statistically analyzed using SPSS version 20.0 (IBM, New York, NY, USA).

\section{Publication of results}

The results of the study will be published at ClinicalTrials. gov and in peer-reviewed medical journals as well. Moreover, we will share our results at scientific meeting in the type of oral presentation or poster presentation.

\section{Discussion}

Real-time ENB has been proved to be an effective tool in the diagnosis of PPLs, while currently commercially available ENB system is used only in conjunction with 

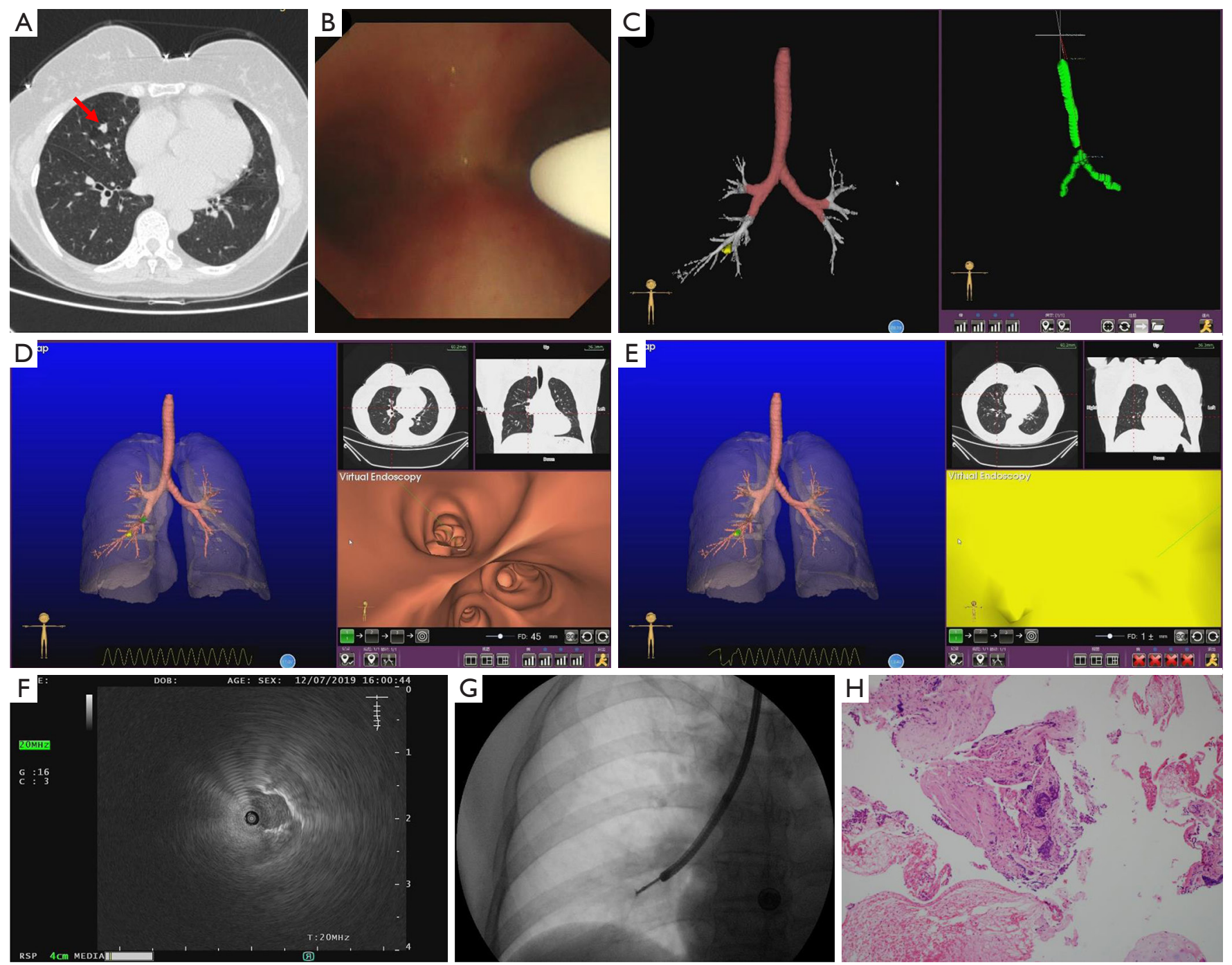

Figure 4 A representative case of the real-word study. A 56 years old female patient with a small solid pulmonary nodule underwent transbronchial biopsy with the guidance of ENB combined with R-EBUS, GS, and fluoroscopy. The HE staining pathology showed small cell lung cancer combined with immunohistochemical staining. (A) Chest CT showed a small pulmonary nodule with the long diameter of $8.3 \mathrm{~mm}$ (red arrow) in the right middle lobe; (B) registration under the bronchoscopy; (C) panel of the ENB system when the registration finished; (D) panel of the ENB system in the process of real-time navigation; (E) panel of the ENB system when the LW reaching the target nodule, (F) R-EBUS image; (G) biopsy with the guidance of fluoroscopy; $(\mathrm{H}) \mathrm{HE}$ staining with the magnification of 10x. ENB, electromagnetic navigation bronchoscopy; R-EBUS, radial probe endobronchial ultrasound; GS, guide sheath; HE, hematoxylin-eosin; CT, computed tomography; LW, locatable wire.

relatively thick EWC $(2.6 \mathrm{~mm})$ and LG $(1.9 \mathrm{~mm})(10,16)$. It is lack of ENB system combined with the commonly used GS of different diameters (2.55 and $1.95 \mathrm{~mm}$ ). The two prospective studies are designed to prove that the innovative ENB system is capable of using thick and thin GS for sampling PPLs, which can improve the diagnostic yield and can save duration time for finding PPLs.
Meta-analyses of ENB in diagnosing PPLs ranges from $55.7 \%$ to $87.5 \%$ (10) and revealed the diagnostic yield seemed to be similar to other guided bronchoscopy procedures, such as the virtual bronchoscopic navigation (VBN) and R-EBUS $(11,19)$. Previous study indicated that thin bronchoscope could reach further into the distal bronchus than conventional bronchoscope (20). There 
have been many studies showing the usefulness of thin or ultrathin bronchoscopes for sampling PPLs combined with VBN $(18,21)$. Oki and his colleagues conducted two studies demonstrating that the combination of an ultrathin bronchoscope (outer diameter $3.0 \mathrm{~mm}$, inner working channel $1.7 \mathrm{~mm}$ ) and a $1.4 \mathrm{~mm}$ mechanical R-EBUS seemed to provide better results than a thin bronchoscope (outer diameter $4.0 \mathrm{~mm})(17,22)$. These studies mentioned above proved that the combination of a thin or ultrathin bronchoscope and navigation device can provide the best combination to introduce biopsy instruments correctly into the leading bronchus and then to PPLs. However, there is no study about ENB combined with thin or ultrathin bronchoscopy at present. This innovative navigation system utilizes a LW of 1.45 or $1.15 \mathrm{~mm}$, which can combine with thin or ultrathin bronchoscope and is able to navigate to very small peripheral airways under direct visualization accurately.

Enrollment for the study began in July 2018 and is currently in progress. In study one, we design two groups to verify the usefulness of the ENB system in diagnosing pulmonary nodules. Then we design a real-world study to explore the optimal combination techniques of the utilization of ENB and the optimal target lesion using ENB. In addition, we can get the diagnostic yield of the novel navigation system in diagnosing pulmonary nodules through the two studies.

With up to 1,150 subjects planned, the two studies we designed will be the largest ENB study of the innovative system to date. The intent of the studies is to assess the usefulness of the novel ENB system and explore the optimal way to use this system through the utilization patterns in the real-world. The two studies will provide a wealth of information regarding the full profile of this new ENB system usage. In addition, clinical utility and outcomes identified in the studies will be instructive for the design of future comparative studies.

\section{Acknowledgments}

Funding: This work was supported by National Key R\&D Program of China (grant number 2017YFC0112700).

\section{Footnote}

Conflicts of Interest: The authors have no conflicts of interest to declare.

Ethical Statement: The authors are accountable for all aspects of the work in ensuring that questions related to the accuracy or integrity of any part of the work are appropriately investigated and resolved. The protocols have been approved by local ethics committee (KS1829) and were registered under ClinicalTrials.gov (NCT03569306 and NCT03716284).

\section{References}

1. Torre LA, Bray F, Siegel RL, et al. Global cancer statistics, 2012. CA Cancer J Clin 2015;65:87-108.

2. Chen $W$, Zheng R, Baade PD, et al. Cancer statistics in China, 2015. CA Cancer J Clin 2016;66:115-32.

3. National Lung Screening Trial Research Team, Aberle DR, Adams AM, et al. Reduced lung-cancer mortality with low-dose computed tomographic screening. $\mathrm{N}$ Engl J Med 2011;365:395-409.

4. Bai C, Choi CM, Chu CM, et al. Evaluation of pulmonary nodules: clinical practice consensus guidelines for Asia. Chest 2016;150:877-93.

5. Gould MK, Donington J, Lynch WR, et al. Evaluation of individuals with pulmonary nodules: when is it lung cancer? Diagnosis and management of lung cancer, 3rd ed: American College of Chest Physicians evidence-based clinical practice guidelines. Chest 2013;143:e93S-120S.

6. Sun J, Xie F, Zheng X, et al Learning curve of electromagnetic navigation bronchoscopy for diagnosing peripheral pulmonary nodules in a single institution. Transl Cancer Res 2017;6:541-51.

7. Schreiner AM, Jones JG, Swistel AJ, et al. Transthoracic fine needle aspiration resulting in implantation metastasis in the superficial tissues of the breast. Cytopathology 2013;24:58-60.

8. Rivera MP, Mehta AC, Wahidi MM. Establishing the diagnosis of lung cancer: diagnosis and management of lung cancer, 3rd ed: American College of Chest Physicians evidence-based clinical practice guidelines. Chest 2013;143:e142S-65S.

9. Ozgul G, Cetinkaya E, Ozgul MA, et al. Efficacy and safety of electromagnetic navigation bronchoscopy with or without radial endobronchial ultrasound for peripheral lung lesions. Endosc Ultrasound 2016;5:189-95.

10. Gex G, Pralong JA, Combescure C, et al. Diagnostic yield and safety of electromagnetic navigation bronchoscopy for lung nodules: a systematic review and meta-analysis. Respiration 2014;87:165-76.

11. Steinfort DP, Khor YH, Manser RL, et al. Radial probe endobronchial ultrasound for the diagnosis of peripheral 
lung cancer: systematic review and meta-analysis. Eur Respir J 2011;37:902-10.

12. Eberhardt R, Ernst A, Herth FJ. Ultrasound-guided transbronchial biopsy of solitary pulmonary nodules less than 20 mm. Eur Respir J 2009;34:1284-7.

13. Eberhardt R, Anantham D, Herth F, et al. Electromagnetic navigation diagnostic bronchoscopy in peripheral lung lesions. Chest 2007;131:1800-5.

14. Ost DE, Ernst A, Lei X, et al. Diagnostic yield and complications of bronchoscopy for peripheral lung lesions. Results of the AQuIRE registry. Am J Respir Crit Care Med 2016;193:68-77.

15. Sun J, Yi X, Zhang X, et al. Diagnostic value of electromagnetic navigation bronchoscopy with a guide sheath for peripheral pulmonary lesions: a randomized controlled trial. Denver: 16th World Conference on Lung Cancer, 2015.

16. Becker HD, Herth F, Ernst A, et al. Bronchoscopic biopsy of peripheral lung lesions under electromagnetic guidance: a pilot study. J Bronchology Interv Pulmonol 2005;12:9-13.

Cite this article as: Xie F, Zhang J, Cao L, Zheng X, Chen J, Li Y, Han B, Sun J. Design of a prospective, multicenter, and cohort study of an innovative electromagnetic navigation bronchoscopy in diagnosing pulmonary nodules among Chinese population. J Thorac Dis 2019;11(12):5592-5600. doi: 10.21037/jtd.2019.11.75
17. Oki M, Saka H, Ando M, et al. Ultrathin bronchoscopy with multimodal devices for peripheral pulmonary lesions. A randomized trial. Am J Respir Crit Care Med 2015;192:468-76.

18. Ishida T, Asano F, Yamazaki K, et al. Virtual bronchoscopic navigation combined with endobronchial ultrasound to diagnose small peripheral pulmonary lesions: a randomised trial. Thorax 2011;66:1072-7.

19. Asano F, Eberhardt R, Herth FJ. Virtual bronchoscopic navigation for peripheral pulmonary lesions. Respiration 2014;88:430-40.

20. Iyoda A, Suzuki M, Chiyo M, et al. A new thin-type bronchoscope improves diagnostic accuracy of peripheral pulmonary carcinoma. Oncol Rep 2003;10:387-9.

21. Asano F, Shinagawa N, Ishida T, et al. Virtual bronchoscopic navigation combined with ultrathin bronchoscopy. A randomized clinical trial. Am J Respir Crit Care Med 2013;188:327-33.

22. Oki M, Saka H, Asano F, et al. Use of an ultrathin vs thin bronchoscope for peripheral pulmonary lesions: a randomized trial. Chest 2019;156:954-64. 